OPEN ACCESS

Edited by:

Kwong Tsang,

Precision Biologics, Inc., United States

Reviewed by:

Pierpaolo Correale,

Azienda ospedaliera 'Bianchi-

Melacrino-Morelli', Italy

Cirino Botta,

University of Palermo, Italy

*Correspondence:

Ilyas Sahin

i.sahin@ufl.edu

Specialty section:

This article was submitted to

Cancer Molecular Targets

and Therapeutics,

a section of the journal

Frontiers in Oncology

Received: 25 October 2021 Accepted: 22 November 2021 Published: 08 December 2021

Citation:

Ozer M, George A, Goksu SY, George TJ and Sahin I (2021) The

Role of Immune Checkpoint Blockade in the Hepatocellular Carcinoma:

A Review of Clinical Trials.

Front. Oncol. 11:801379.

doi: 10.3389/fonc.2021.801379

\section{The Role of Immune Checkpoint Blockade in the Hepatocellular Carcinoma: A Review of Clinical Trials}

\author{
Muhammet Ozer ${ }^{1}$, Andrew George ${ }^{2,3}$, Suleyman Yasin Goksu ${ }^{4}$, \\ Thomas J. George ${ }^{5,6}$ and Ilyas Sahin ${ }^{5,6 *}$
}

${ }^{1}$ Department of Internal Medicine, Capital Health Medical Center, Trenton, NJ, United States, ${ }^{2}$ Department of Chemistry, Brown University, Providence, RI, United States, ${ }^{3}$ Department of Molecular Biology, Cell Biology \& Biochemistry, Division of Biology and Medicine, Brown University, Providence, RI, United States, ${ }^{4}$ Division of Hematology/Oncology, Department of Medicine, University of Texas Southwestern Medical Center, Dallas, TX, United States, ${ }^{5}$ Division of Hematology/Oncology, Department of Medicine, University of Florida, Gainesville, FL, United States, ${ }^{6}$ Division of Hematology/Oncology, Department of Medicine, University of Florida Health Cancer Center, Gainesville, FL, United States

The prevalence of primary liver cancer is rapidly rising all around the world. Hepatocellular carcinoma $(\mathrm{HCC})$ is the most common type of primary liver cancer. Unfortunately, the traditional treatment methods to cure HCC showed poor efficacy in patients who are not candidates for liver transplantation. Until recently, tyrosine kinase inhibitors (TKIs) were the front-line treatment for unresectable liver cancer. However, rapidly emerging new data has drastically changed the landscape of HCC treatment. The combination treatment of atezolizumab plus bevacizumab (immunotherapy plus anti-VEGF) was shown to provide superior outcomes and has become the new standard first-line treatment for unresectable or metastatic HCC. Currently, ongoing clinical trials with immune checkpoint blockade (ICB) have focused on assessing the benefit of antibodies against programmed cell death 1 (PD-1), programmed cell death-ligand 1 (PD-L1), and cytotoxic T-lymphocyteassociated antigen 4 (CTLA-4) as monotherapies or combination therapies in patients with HCC. In this review, we briefly discuss the mechanisms underlying various novel immune checkpoint blockade therapies and combination modalities along with recent/ ongoing clinical trials which may generate innovative new treatment approaches with potential new FDA approvals for HCC treatment in the near future.

Keywords: immune checkpoint blockade, immunotherapy, hepatocellular carcinoma (HCC), liver cancer (LC), clinical trials

\section{INTRODUCTION}

Hepatocellular carcinoma (HCC) is a primary malignant disease of the liver. Primary liver cancer is the seventh most common malignancy and the second most frequent cancer-related death worldwide (1). The majority of HCC patients are diagnosed with unresectable or advanced disease and have a poor prognosis (2). Thus, clinicians and scientists are eager to find novel 
therapeutic approaches for HCC that effectively balance clinical benefit with toxicity. The main objective for early stage HCC is curing the disease, and traditional therapeutic methods mainly include surgery, liver translaplantation or other localized liverdirected therapies (e.g., trans-arterial chemoembolization [TACE] and radiofrequency ablation [RFA]). However, treatment options for the advanced stage HCC are relatively limited and palliative in nature (3).

Until recently, tyrosine-kinase inhibitors (TKIs) like oral sorafenib were the most common gold-standard treatment for advanced HCC. However, the IMbrave150 trial (NCT03434379) demonstrated that atezolizumab combined with bevacizumab resulted in better overall survival (OS) and progression-free survival (PFS), effectively replacing sorafenib as a new standard first-line treatment for advanced HCC, particularly for those with near normal liver function (4). In situations where atezolizumab with bevacizumab is contraindicated or not available, TKIs (preferably sorafenib or lenvatinib) may also be considered for first-line treatment of advanced HCC. Otherwise, alternative treatments including nivolumab and other agents with less data supporting their use may be considered, consistent with national guidelines (3). As second-line treatments, regorafenib and cabozantinib were shown to prolong survival compared with placebo and received approval from the United States Food and Drug Administration (FDA) for advanced HCC patients who previously received sorafenib $(5,6)$. Ramucirumab also showed efficacy after sorafenib among advanced HCC patients with AFP levels $>400 \mathrm{ng} / \mathrm{ml}$ (7). Although the prognosis of HCC remains dismal, the recent developments in systemic therapies - particularly in immunotherapy and targeted therapies - have increased overall survival and the quality of life of patients.

In the near past, oncolytic immunotherapy has arisen as a promising approach for inhibiting tumor progression and metastasis (8). The justification of this approach relates to enhancing cellular or humoral immunity via activating tumorspecific immune responses and disrupting immune tolerance. Developments in this field have led to many FDA approvals of immune checkpoint inhibitors (ICI) as primary treatment options for several different solid and hematologic malignancies (9). Moreover, novel treatment combinations along with newly identified druggable targets are expected to expand the role of immunotherapy in the treatment of a variety of cancers in the coming years. Recent developments also suggest promising antitumor effects of immunotherapy in HCC, highlighting the importance of this treatment modality amongst an otherwise limited set of treatment options.

Immune checkpoint molecules mainly function to maintain immunotherapeutic balance and protect against uncontrolled immunity by preventing excessive activation of $\mathrm{T}$ cells (10). However, negative immune regulators may be overexpressed in tumors to escape immune surveillance. Therapeutic ICIs counteract this escape and reactivate tumor-specific $\mathrm{T}$ cells via suppressing checkpoint-mediated signals (11). Cytotoxic Tlymphocyte-associated antigen 4 (CTLA-4), programmed cell death protein 1 (PD-1), programmed cell death protein-ligand 1
(PD-L1), B and T lymphocyte attenuator (BTLA), V-domain immunoglobulin suppressor of $\mathrm{T}$ cell activation (VISTA), T-cell immunoglobulin and mucin domain 3 (TIM-3), lymphocyte activation gene-3 (LAG-3), and tumor necrosis factor receptor superfamily member 4 (OX40) are the main ICIs under investigation (12). Although it is a promising treatment modality for a variety of cancers, tumors often exhibit primary, adaptive, or acquired resistance to immunotherapy which might be intrinsic to the tumor cells or that may be influenced by their microenvironment (13). In addition to cytotoxic $\mathrm{T}$ cells, there are other essential mediators of immune homeostasis such as $\mathrm{T}$ regulatory cells (Tregs) (14), myeloid-derived suppressor cells (MDSCs) (15), regulatory dendritic cells (16), and NK cells (17) which also play an essential role in response to immunotherapy treatments.

The conditional FDA approval in 2017 of the anti-PD-1 antibody nivolumab to treat advanced HCC in patients who have been previously treated with sorafenib opened a new era of drug development for advanced HCC. Single-agent ICIs were found to provide clinical benefits in $15-20 \%$ of responders, however, biomarkers have failed to help identify this subgroup (18). Fortunately, there are multiple early and advanced stage clinical trials investigating the efficacy of combination therapies, including combining ICIs with TKIs or combining PD1/PDL1 axis inhibitors with CTLA4 inhibitors, which might change the landscape of HCC management for different stages in the near future. Current research progress in the treatment of HCC mainly include ICIs, tumor vaccines, and adoptive cell therapy. This paper reviews ongoing clinical trials of ICIs in HCC patients.

\section{BACKGROUND OF IMMUNE CHECKPOINT INHIBITORS (ICIS) IN HCC}

Tasuku Honjo identified PD-1 as an immune checkpoint molecule at Kyoto University in 1992 (19). Many years later, nivolumab targeting the PD-1 was approved for the treatment of patients with melanoma in 2014 as the first anti-PD-1 antibody. Consequently, the FDA granted approval to nivolumab for the treatment of non-small-cell lung cancer and kidney cancer in the USA. James Allison was the first to show that CTLA-4 is also a therapeutic target for cancer treatment (20). Ipilimumab, an antibody targeting CTLA-4, was subsequently developed and approved by the FDA as an anti-melanoma agent in the USA in 2011 (21). Together, these two pioneers were each awarded the Nobel Prize in Physiology or Medicine in 2018, reflecting the impact of their seminal work on the field of oncology (22).

The liver has a highly complex immune tolerance system driven by antigen-presenting cells (APCs), namely dendritic cells (DCs) and liver-specific APCs. These complex antigen presentation mechanisms require multiple costimulatory signals to achieve T-cell activation and clonal expansion. Meanwhile, APCs also produce additional signals for immune checkpoint molecules, limiting $\mathrm{T}$ cell hyperactivation. These suppressive signals from immune checkpoint molecules have 
an active role in maintaining tolerance and preventing unwanted immune responses. Malignant tumor environments disrupt normal suppressive signals and cause T-cell exhaustion, characterized by high expression of immune checkpoint molecules, impaired cytotoxicity, and low levels of effector cytokines, leading to chronic hyporesponsive immunity (23). Since immune checkpoint molecules seem responsible for immunotolerance, many clinical trials have been designed to confirm their function in HCC treatment.

PD-1 is mainly expressed in T cells, B cells, natural killer (NK) cells, mononuclear cells, and dendritic cells (24). PD-1 inhibitors activate immune cells by blocking the receptor binding of PD-L1 and PD-L2 (25). Well-known PD-1 inhibitors nivolumab and pembrolizumab have largely been investigated for immunotherapy in cancer patients. A non-comparative phase I/II CheckMate 040 trial of Nivolumab (NCT01658878) with 262 patients with advanced HCC showed the safety and efficacy of PD-1 inhibitor in treating HCCs (26). The objective response rate (ORR) was $20 \%$, with a median OS of 15 months. Nivolumab activity was observed regardless of tumor PD-L1 expression, prior sorafenib exposure, and underlying etiology. Based on this study, in 2017, nivolumab received conditional approval as a second-line treatment of patients with advanced and metastatic HCC. To evaluate nivolumab further as a monotherapy, an open-label, multicenter, randomized Phase III Checkmate 459 trial comparing nivolumab with sorafenib (NCT02576509) as first-line therapy was conducted in patients with advanced HCC (27). At ESMO World Congress on Gastrointestinal Cancer 2020 Virtual, the study team presented long-term follow-up results. Although 1.5 months longer OS was observed with nivolumab, it did not meet the predefined threshold for significance with a minimum follow-up of 33.6 months. However, nivolumab had a more favorable and manageable safety profile with better preservation of liver function over time compared with sorafenib. Another anti-PD1 antibody, pembrolizumab, has been studied in a multicenter, randomized Phase II KEYNOTE-224 study (NCT02702414) in sorafenib-refractory advanced HCC patients (28). The ORR was $17 \%$, with a $1 \%$ complete response (CR), $16 \%$ partial responses (PR). Stable disease (SD) was achieved in $44 \%$ of patients, the median OS was 12.9 months, and the PFS was 4.9 months (28). With those results, the FDA approved pembrolizumab as a second-line agent in patients with HCC whose disease progressed under sorafenib treatment. The subsequent randomized, double-blind Phase III KEYNOTE-240 (NCT02702401) assessed pembrolizumab versus placebo in the second-line setting. Unfortunately, the HR for OS $(0.781 ; 95 \%$ CI, 0.611 to 0.998 ; P 5.0238) and PFS (HR, 0.72; 95\% CI, 0.57 to 0.90) did not reach prespecified statistical plan (29).

Anti-PD-L1 antibodies, durvalumab and avelumab, are currently undergoing active assessment in clinical trials. Durvalumab showed $10.3 \%$ ORR in Phase I/II study of advanced HCC patients following prior treatment with sorafenib (NCT01693562) (30). In a single-arm phase II study, avelumab was evaluated in patients with advanced HCC also previously treated with sorafenib (NCT03389126). After a median follow-up of 13.7 months, avelumab showed moderate efficacy (no CR, 10.0\% PR, and 63.3\% SD) and was well tolerated (no grade 4 adverse events and 23\% grade 3 adverse events) (31). Other PD-1/PD-L1 trials, including tislelizumab (NCT03412773, NCT03419897) are currently in development as well.

CTLA-4 is mainly expressed by Tregs, activated T cells, and NK cells (32). CTLA-4 inhibitors accelerate T cell activation by preventing the binding of CTLA-4 to B7-1 and B7-2. One of the well-known actions of the anti-CTLA-4 antibody is to downregulate Tregs in the tumor microenvironment. Given that CTLA-4 is most strongly expressed on Tregs, the mechanism of action of anti- CTLA-4 antibodies may involve inhibition of Tregs activity. As the earliest immune checkpoint inhibitor therapy, a phase II study of the anti-CTLA-4 antibody tremelimumab (NCT01008358) showed a promising effect with a $17.6 \%$ partial response rate, and a $76 \%$ disease control rate in patients with advanced HCC whose disease progressed under sorafenib treatment (33). Current ongoing clinical studies are actively investigating the efficacy and safety of the anti-CTLA-4 antibodies ipilimumab and tremelimumab. Many ongoing clinical trials of monotherapy for ICIs in HCC are listed in Table 1.

\section{COMBINATION THERAPY WITH IMMUNE CHECKPOINT INHIBITORS}

Combination therapies with ICIs are a rising trend due to the modest activity of monotherapies in this disease. A combination of ICIs with multi-TKIs, conventional ablative therapies, or other immune modulating agents represent the next generation of HCC treatment modalities currently undergoing active investigation (Figure 1). Preclinical studies showed the potential synergistic efficacy of ICIs with TKIs and antiangiogenic drugs. Immunosuppressive properties of vascular endothelial growth factor (VEGF) have been demonstrated in preclinical models via inhibition of T-cell infiltration due to diminished permeability of tumor vessels $(34,35)$. Additionally, VEGF inhibits T-cell development and causes the upregulation of PD-1 and CTLA-4 expression on immune cells $(36,37)$. Although the clinical activity as single agent is modest, it has been shown that anti-angiogenic drugs could normalize the tumor vasculature and increase $\mathrm{T}$ cell infiltration by inducing the upregulation of the leukocyte adhesion molecules such as ICAM-1 and VCAM-1 on tumor endothelial cells (34). It was previously shown that VEGF inhibitor could synergize with ICIs in metastatic melanoma and there is an increase interest in combining anti-angiogenic treatments with ICIs to turn "cold' tumors into "hot" tumors (38). Among other TKIs, cabozantinib's potential effect of enhancing the response to ICIs (39) and lenvatinib's immunemodulating activity when combined with PD-1 inhibitor were previously shown (40).

Recently, open-label, phase 3 IMbrave150 (NCT03434379) trial compared the efficacy of atezolizumab plus bevacizumab combination with sorafenib therapy in first line setting in 501 
TABLE 1 | Ongoing clinical trials of monotherapy for immune checkpoint inhibitors in hepatocellular carcinoma (HCC).

\begin{tabular}{|c|c|c|c|c|c|}
\hline Agent & Phase & Clinicaltrial.gov & Trial Name & Patient characteristics & $\begin{array}{l}\text { Primary } \\
\text { Outcome }\end{array}$ \\
\hline Nivolumab & Phase 2 & NCT03630640 & NIVOLEP & Neoadjuvant and adjuvant treatment in patients treated by Electroporation & Local RFS \\
\hline Nivolumab & Phase 3 & NCT03383458 & $\begin{array}{l}\text { CheckMate } \\
\text { 9DX }\end{array}$ & Patients who received curative resection or ablation & RFS \\
\hline Nivolumab & $\begin{array}{l}\text { Phase } 1 \& \\
2\end{array}$ & NCT01658878 & $\begin{array}{l}\text { CheckMate- } \\
040\end{array}$ & Systemic treatment naïve patients with advanced HCC & ORR, AEs \\
\hline Pembrolizumab & Phase 1 & NCT02595866 & - & Patients with HIV and relapsed, refractory or disseminated HCC & AEs \\
\hline Pembrolizumab & Phase 2 & NCT02702414 & KEYNOTE-224 & Patients with advanced HCC & ORR \\
\hline Pembrolizumab & Phase 2 & NCT03163992 & - & $2^{\text {nd }}$ line treatment after Sorafenib failure & ORR \\
\hline Pembrolizumab & Phase 3 & NCT03867084 & KEYNOTE-937 & $\begin{array}{l}\text { Patients with HCC who has complete radiologic response after resection or local } \\
\text { ablation }\end{array}$ & RFS, OS \\
\hline Pembrolizumab & $\begin{array}{l}\text { Phase } 1 \& \\
2\end{array}$ & NCT02940496 & - & $2^{\text {nd }}$ line treatment in advanced $\mathrm{HCC}$ & DLTS \\
\hline Pembrolizumab & Phase 2 & NCT03419481 & - & Hep B related HCC & $\mathrm{RR}$ \\
\hline Pembrolizumab & Phase 3 & NCT03062358 & KEYNOTE-394 & $2^{\text {nd }}$ line treatment in Asian patients & OS \\
\hline Toripalimab & $\begin{array}{l}\text { Phase } 2 \text { \& } \\
3\end{array}$ & NCT03859128 & JUPITER-04 & Adjuvant therapy following radical resection & RFS \\
\hline Tislelizumab & Phase 3 & NCT03412773 & $\begin{array}{l}\text { RATIONALE- } \\
301\end{array}$ & $1^{\text {st }}$ line treatment in patients with advanced HCC & OS \\
\hline Tislelizumab & Phase 2 & NCT03419897 & $\begin{array}{l}\text { RATIONALE- } \\
208\end{array}$ & Patients who have been previously systemically treated & ORR \\
\hline
\end{tabular}

patients with advanced HCC. Atezolizumab plus bevacizumab showed significantly better OS at 12 months than sorafenib (67.2\% vs. $54.6 \%$ respectively). Also, median PFS was 6.8 months in the combination arm while 4.3 months in sorafenib group (hazard ratio for disease progression or death, 0.59 ; $95 \%$ CI, 0.47 to 0.76 ; $\mathrm{P}<0.001$ ) (41). Also, combination therapies targeting PD-1/PD-L1 and CTLA-4 are very effective in patients with malignant melanoma $(42,43)$, microsatellite instability high colorectal cancer, and are being investigated in many ongoing clinical trials in patients with HCC (44). The rationale for this strategy is based on the hypothesis that if CD8-positive $\mathrm{T}$ cells do not exist in cancer cells, blockade of the PD-1/PD-L1 pathway's effectivity will be lower than expected. For this reason, blockade of the CTLA-4 can be an effective strategy to enhance the number of activated CD8-positive $\mathrm{T}$ cells that infiltrate the tumor cells. Based on preclinical data, many studies are currently evaluating the effectiveness of combination therapy with inhibitors against CTLA-4, PD-1, and PD-L1 in HCC (Table 2). The phase I/II CheckMate 040 trial (NCT01658878) investigated the effectiveness of the combination therapy of nivolumab and ipilimumab for the first time using various doses. On March 10, 2020, the FDA granted accelerated approval to the combination of nivolumab plus ipilimumab treatment for the patients with advanced HCC who have been previously treated with sorafenib. He et al. presented the results in the 2020 American Society of Oncology Gastrointestinal Cancers Symposium (ASCO-GI) Annual Meeting (45). The FDA approved the combination treatment based on promising ORR and duration of response (DOR). After a median follow-up of 30.7 months, $32 \%(16 / 49)$ of patients responded to combination treatment, with $8 \%(4 / 49)$ having a CR and $24 \%$ (12/49) having a PR (46). To date, this is the only dual immunotherapy approved by the FDA in the second-line setting treatment of HCC. Combination therapy with durvalumab and tremelimumab is being evaluated in a few ongoing clinical trials. An open-labeled, randomized phase I/II study (NCT02519348) testing this combination in patients with unresectable HCC appears safe and tolerable with a disease control rate of $57.5 \%$ (23/40 patients) and confirmed response rate of $15 \%$ (6/40 patients) (47). However, these promising results require further evaluation in a randomized setting and a larger patient population. Thus, an open-label, randomized, multicenter phase III clinical trial (NCT03298451) is being conducted to assess the efficacy and tolerability of the durvalumab plus tremelimumab combination therapy versus sorafenib as first-line treatment of patients with unresectable advanced HCC with results eagerly awaited.

TKIs and immune checkpoint inhibitors separately have proven efficacy in treating HCC. Thus, combined TKIs and immune checkpoint inhibitors have been investigated actively in recent clinical trials to obtain a better survival rate. Kudo et al. published results from a phase $1 \mathrm{~b}$ study of avelumab plus axitinib as first-line treatment in patients with advanced HCC (NCT03289533). They reported that the avelumab plus axitinib combination showed antitumor activity (ORR: 31.8\% [95\% CI: 13.9-54.9\%] per mRECIST for HCC) and was associated with a manageable toxicity profile (48). Other combination treatments of nivolumab plus sorafenib and nivolumab plus cabozantinib are under evaluation in phase I/II clinical studies (NCT03439891, NCT03299946). Combinations of pembrolizumab with either lenvatinib or regorafenib are similarly under evaluation in early-stage clinical trials. In vitro and in vivo preclinical studies of pembrolizumab plus lenvatinib combinations decreased TGF- $\beta$ and IL- 10 by suppressing tumorassociated macrophages, Tregs, and related tumor microenvironment constituents (49). Preclinical work also demonstrated an effect on the downregulation of PD-1 and TIM3. Similar future studies are needed to identify the clear 


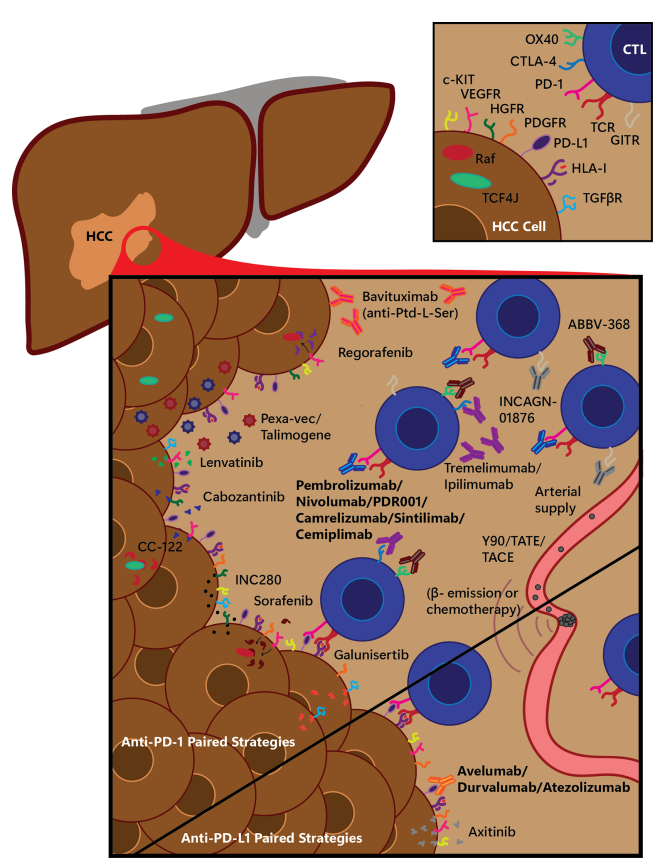

FIGURE 1 | Selected experimental immunotherapy treatment combination strategies for anti-PD-1 and anti-PD-L1 therapy. Many small molecule drugs and other treatments are being tested in combination with standard immune checkpoint blockade (ICB) immunotherapeutics; a graphical overview of a selection of these are shown here, being tested with anti-PD-1 or anti-PD-L1 therapies. Main figure depicts various treatment strategies on a hepatocellular carcinoma (HCC) tumor, infiltrating cytotoxic T-lymphocytes (CTLs), and arterial-derived tumor-specific vasculature, divided into treatments being tested along with anti-PD-1 therapy (top, tested with either Pembrolizumab, Nivolumab, or PDR001) or with anti-PD-L1 therapy (bottom, tested with Avelumab, Durvalumab or Atezolizumab). $\beta$-, electron emission radiation; CTL, cytotoxic T-lymphocyte; CTLA-4, cytotoxic-T-lymphocyte-associated protein 4; HCC, hepatocellular carcinoma; HGFR, hepatocyte growth factor receptor; HLA-I, human leukocyte antigen class I; GITR, glucocorticoid-induced tumor necrosis factor receptor related; PD-1, programmed cell death protein 1; PDL1, programmed cell death protein ligand 1; PDGFR, platelet-derived growth factor receptor; Pexa-Vec, pexastimogene devacirepvec; Ptd-L-Ser, phosphatidylserine; TACE, trans-arterial chemoembolization; TATE, transarterial tirapazamine embolization; TCF4J, transcription factor $4 \mathrm{~J}$ isoform; TCR, T-cell receptor; TGF $\beta$ R, transforming growth factor $\beta$ receptor; VEGFR, vascular endothelial growth factor receptor; Y90, yttrium-90 radioembolization.

effects of the combined modalities of TKIs and immune checkpoint inhibitors. Ongoing combination therapies with ICIs and TKIs in clinical trials are summarized in Table 2.

Patients who received immunotherapy may develop immunerelated adverse events (irAEs) due to their critical roles in selftolerance. While a general perception is that ICI related to toxicities are milder than traditional cytotoxic and molecular targeted anticancer agents, some ICI-induced autoimmune conditions can be lethal, particularly if not immediately recognized (50). The most common immune-related adverse events in HCC patients who underwent anti-CTLA-4 treatment are skin rash, fatigue, and diarrhea (33). Treatment with
anti-PD-1 antibodies causes fewer immune-related adverse events in patients with HCC than anti-CTLA-4 therapies. Reactivation of T-cells can cause cytotoxicity and an increase in hepatic damage. Withdrawal of ICI treatment and initiation of steroid therapy can control the vast majority of immune-related adverse events. There are well-established practical guidelines for evaluation and optimization of irAE management, including in populations of special interest $(51,52)$.

Second-generation immunotherapies such as TIM-3, LAG-3, and killer cell immunoglobulin-like receptor (KIR) are still in the relatively early stages of clinical development for advanced solid tumors (53). Combining these with anti-PD-1 antibody therapies represent novel ways to induce $\mathrm{T}$-cell infiltration into the tumor microenvironment. Combinations of anti-PD1 therapies with LAG-3 (NCT03005782 and NCT01968109) and TIM-3 (NCT03099109) are currently in phase 1 clinical trials. Additional clinical trials will continue to assess the efficacy and safety of novel ICIs in treating advanced HCC.

\section{IMMUNE RELATED ADVERSE EVENTS OF COMBINATION IMMUNOTHERAPIES}

With the development of cancer immunotherapy, the combination strategies are becoming prevalent. Moreover, immune-related adverse events (irAEs) occurred more frequently, showing a different pattern from single-agent therapy. In recent years, anti-PD-1 and anti-CTLA-4 antibodies combinations have been extensively studied, showing higher response rates than single-agent therapy. Previous studies showed that both the incidence and severity of irAEs increase with the combination of anti-CTLA-4 and antiPD-1 therapy (54-56). A phase 3 CheckMate067 study showed that the incidence of grade 3 or 4 irAEs was $59 \%$ in the nivolumab plus ipilimumab combination group, $28 \%$ in the ipilimumab group $21 \%$ in the nivolumab group. The most common grade 3/4 irAEs were gastrointestinal reactions. Similarly, the Checkmat040 study showed an increased risk of irAEs in advanced HCC patients (57). Anti-PDL-1 plus antiCTLA-4 combination immunotherapy also showed a higher irAEs profile. In a phase, I/II study of durvalumab plus tremelimumab combination and monotherapies tolerability was acceptable across arms. Grade 3 irAEs occurred $37.8 \%$ in combination arm while $20.8 \%$ in durvalumab arm (58). A combination of anti-PD-1 therapy with other immune checkpoint molecules is also under ongoing investigation. Previous studies showed that a combination of nivolumab plus LAG-3 was well tolerated. The most common irAEs were fatigue, diarrhea, and pruritis. Grade 3/4 irAEs occurred in $9 \%$ of patients (59).

To date, there are no immunotherapy combinations related to specific irAEs; however, the incidence of serious irAEs is higher than monotherapies. For grade 2 or above irAES, ASCO, and National Comprehensive Cancer Network (NCCN) practice guidelines suggest treatment interruption, close monitoring, supportive management $(51,60)$. 
TABLE 2 | Ongoing clinical trials of combination therapy for immune checkpoint inhibitors in hepatocellular carcinoma (HCC).

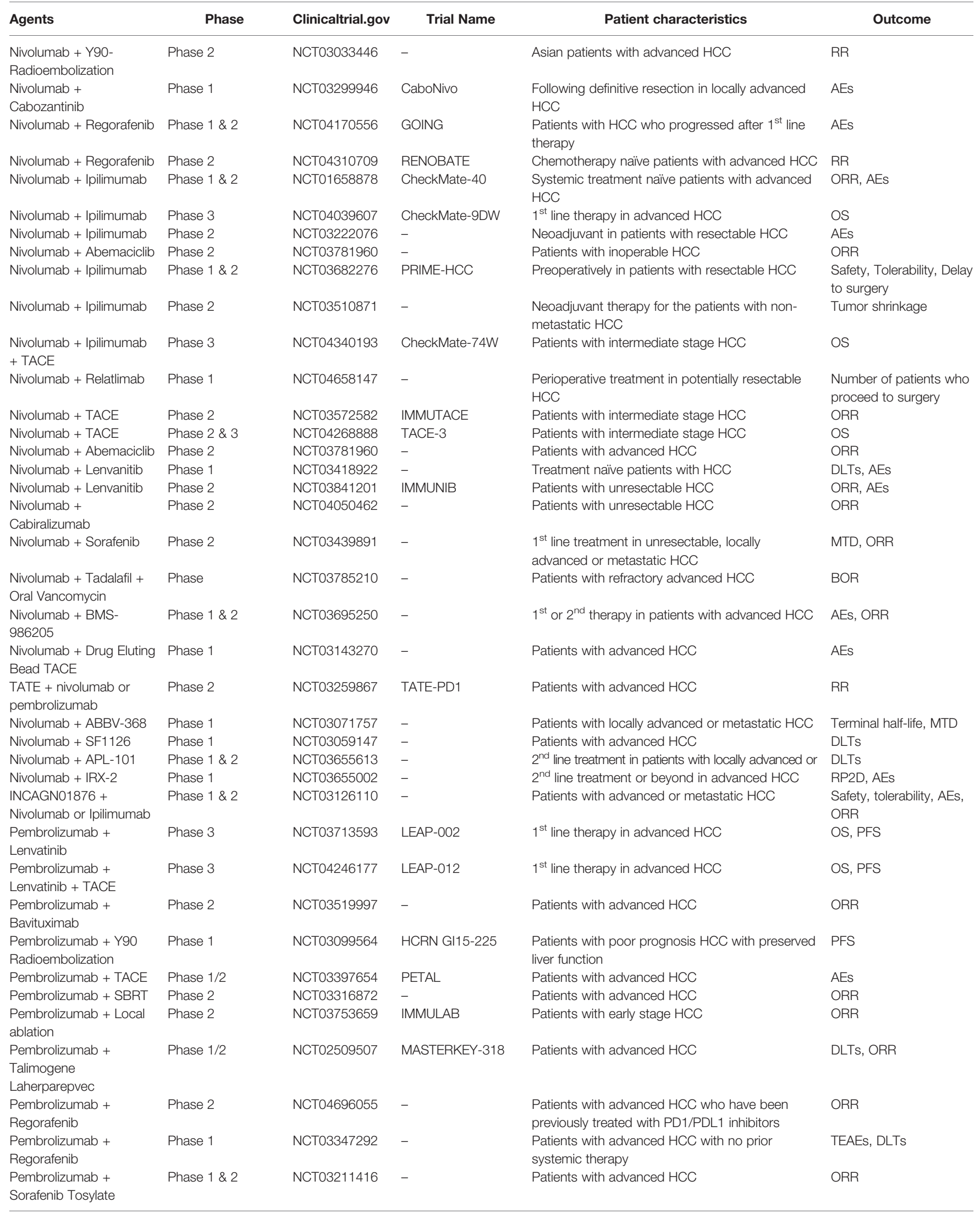


TABLE 2 | Continued

\begin{tabular}{|c|c|c|c|c|c|}
\hline Agents & Phase & Clinicaltrial.gov & Trial Name & Patient characteristics & Outcome \\
\hline $\begin{array}{l}\text { Pembrolizumab + } \\
\text { p53MVA vaccine }\end{array}$ & Phase 1 & NCT02432963 & - & $\begin{array}{l}\text { Patients with solid tumors including HCC that } \\
\text { have failed prior therapy }\end{array}$ & Tolerability \\
\hline $\begin{array}{l}\text { Atezolizumab }+ \\
\text { Cabozantinib }\end{array}$ & Phase 3 & NCT03755791 & COSMIC-312 & $1^{\text {st }}$ line therapy in advanced HCC & PFS, OS \\
\hline $\begin{array}{l}\text { Atezolizumab + } \\
\text { Cabozantinib }\end{array}$ & Phase 1 \& 2 & NCT03170960 & - & $1^{\text {st }}$ line treatment in advanced $\mathrm{HCC}$ & ORR, MTD \\
\hline $\begin{array}{l}\text { Atezolizumab + } \\
\text { Bevacizumab }\end{array}$ & Phase 3 & NCT04102098 & IMbrave050 & $\begin{array}{l}\text { Patients with } \mathrm{HCC} \text { who has high risk of } \\
\text { recurrence after resection or ablation }\end{array}$ & RFS \\
\hline $\begin{array}{l}\text { Durvalumab +/- } \\
\text { Tremelimumab }\end{array}$ & Phase 3 & NCT03298451 & HIMALAYA & $1^{\text {st }}$ line treatment in advanced HCC & OS \\
\hline $\begin{array}{l}\text { Durvalumab +/- } \\
\text { Tremelimumab }\end{array}$ & Phase 2 & NCT02519348 & - & Immunotherapy naïve patients with advanced HCC & AEs, DLTS \\
\hline $\begin{array}{l}\text { Durvalumab + } \\
\text { Tremelimumab }\end{array}$ & Phase 2 & NCT03638141 & - & Patients with intermediate stage $\mathrm{HCC}$ & ORR \\
\hline $\begin{array}{l}\text { Durvalumab + } \\
\text { Cabozantinib }\end{array}$ & Phase 1 \& 2 & NCT02572687 & CAMILLA & $\begin{array}{l}\text { Advanced HCC progressed under at least } 1 \\
\text { previous systemic therapy }\end{array}$ & ORR, MTD \\
\hline $\begin{array}{l}\text { Durvalumab + } \\
\text { Tremelimumab +/- TACE }\end{array}$ & Phase 2 & NCT02821754 & - & $\begin{array}{l}\text { Patients with HCC that progressed or intolerant to } \\
\text { at least one line of therapy }\end{array}$ & PFS \\
\hline $\begin{array}{l}\text { Durvalumab + } \\
\text { Tremelimumab + } \\
\text { Radiation }\end{array}$ & Phase 2 & NCT03482102 & - & $\begin{array}{l}\text { Patients with locally advanced, advanced or } \\
\text { metastatic HCC }\end{array}$ & $\mathrm{BOR}$ \\
\hline $\begin{array}{l}\text { Durvalumab + TACE +/- } \\
\text { Bevacizumab }\end{array}$ & Phase 3 & NCT03778957 & EMERALD-1 & Patients with locoregional HCC & PFS \\
\hline $\begin{array}{l}\text { Durvalumab +/- } \\
\text { Bevacizumab }\end{array}$ & Phase 3 & NCT03847428 & EMERALD-2 & $\begin{array}{l}\text { Patients with HCC who has high risk of } \\
\text { recurrence after curative resection or ablation }\end{array}$ & RFS \\
\hline Camrelizumab + Apatinib & Phase 2 & NCT03463876 & RESCUE & Patients with advanced HCC & ORR \\
\hline Camrelizumab + Apatinib & Phase 3 & NCT03764293 & - & $1^{\text {st }}$ line therapy in advanced HCC & OS, PFS \\
\hline $\begin{array}{l}\text { Camrelizumab + } \\
\text { FOLFOX4 }\end{array}$ & Phase 3 & NCT03605706 & - & $1^{\text {st }}$ line therapy in advanced HCC & OS \\
\hline $\begin{array}{l}\text { Sintilimab }+ \\
\text { Bevacizumab biosimilar }\end{array}$ & Phase 2 \& 3 & NCT03794440 & ORIENT-32 & $1^{\text {st }}$ line therapy in advanced HCC & OS. PFS \\
\hline Anti-LAG-3 + REGN2810 & Phase 1 & NCT03005782 & - & $\begin{array}{l}\text { Patients with advanced solid tumors including } \\
\text { HCC }\end{array}$ & AEs, DLTs \\
\hline Anti-LAG-3 + Nivolumab & Phase 1 \& 2 & NCT01968109 & - & Patients with advanced solid tumors including HCC & AEs, DCR \\
\hline Anti-TIM-3 + LY3300054 & Phase 1 & NCT03099109 & - & $\begin{array}{l}\text { Patients with advanced/relapsed solid tumors } \\
\text { including HCC }\end{array}$ & DLTs \\
\hline
\end{tabular}

\section{CONCLUSION AND FUTURE EXPECTATIONS}

HCC is the second most frequent cause of cancer-related death worldwide, with limited therapeutic options. The immune system has an important and complex role in the pathogenesis of the HCC. Since the discovery of immune checkpoint proteins and development of their inhibitors, the treatment algorithms for HCC have dramatically changed and represent promising opportunities for patients. While ICIs have demonstrated promising results, efficacy remains limited for most patients and future improvements can only come through rigorous

\section{REFERENCES}

1. Sung H, Ferlay J, Siegel RL, Laversanne M, Soerjomataram I, Jemal A, et al. Global Cancer Statistics 2020: GLOBOCAN Estimates of Incidence and Mortality Worldwide for 36 Cancers in 185 Countries. CA Cancer J Clin (2021) 71(3):209-49. doi: 10.3322/caac.21660

2. Llovet JM, Cucreux M, Lencioni R, Di Bisceglie AM, Galle PR, Dufour F. EASL-EORTC Clinical Practice Guidelines: Management of Hepatocellular Carcinoma. J Hepatol (2012) 56(4):908-43. doi: 10.1016/j.jhep.2011.12.001 scientific investigations. We believe that ICIs will play an essential future role in the therapeutic interventions for patients with HCC, with the realization of improved clinical outcomes for all.

\section{AUTHOR CONTRIBUTIONS}

All authors contributed to the article and approved the submitted version. The corresponding author is responsible for submitting a competing financial interest statement on behalf of all authors of the paper. 
Phase 3 Trial. Lancet (2017) 389(10064):56-66. doi: 10.1016/S0140-6736(16) 32453-9

6. Abou-Alfa GK, Meyer T, Cheng AL, El-Khoueiry AB, Rimassa L, Ryoo BY, et al. Cabozantinib in Patients With Advanced and Progressing Hepatocellular Carcinoma. N Engl J Med (2018) 379(1):54-63. doi: 10.1056/ NEJMoa1717002

7. Zhu AX, Kang YK, Yen CJ, Finn RS, Galle PR, Llovet JM, et al. Ramucirumab After Sorafenib in Patients With Advanced Hepatocellular Carcinoma and Increased Alpha-Fetoprotein Concentrations (REACH-2): A Randomised, Double-Blind, Placebo-Controlled, Phase 3 Trial. Lancet Oncol (2019) 20 (2):282-96. doi: 10.1016/S1470-2045(18)30937-9

8. Hernandez-Gea V, Toffanin S, Friedman SL, Llovet JM. Role of the Microenvironment in the Pathogenesis and Treatment of Hepatocellular Carcinoma. Gastroenterology (2013) 144(3):512-27. doi: 10.1053/j.gastro. 2013.01.002

9. Waldman AD, Fritz JM, Lenardo MJ. A Guide to Cancer Immunotherapy: From T Cell Basic Science to Clinical Practice. Nat Rev Immunol (2020) 20 (11):651-68. doi: 10.1038/s41577-020-0306-5

10. Chen Z, Shen S, Peng B, Tao J. Intratumoural GM-CSF Microspheres and CTLA-4 Blockade Enhance the Antitumour Immunity Induced by Thermal Ablation in a Subcutaneous Murine Hepatoma Model. Int J Hyperthermia Off J Eur Soc Hyperthermic Oncol North Am Hyperthermia Group (2009) 25 (5):374-82. doi: 10.1080/02656730902976807

11. Haanen JB, Robert C. Immune Checkpoint Inhibitors. Prog Tumor Res (2015) 42:55-66. doi: 10.1159/000437178

12. Hato T, Goyal L, Greten TF, Duda DG, Zhu AX. Immune Checkpoint Blockade in Hepatocellular Carcinoma: Current Progress and Future Directions. Hepatol (Baltimore Md) (2014) 60(5):1776-82. doi: 10.1002/ hep. 27246

13. George A, Sahin I, Carneiro BA, Dizon DS, Safran HP, El-Deiry WS. Strategies to Sensitize Cancer Cells to Immunotherapy. Hum Vaccin Immunother (2021) 17(8):2595-601. doi: 10.1080/21645515.2021.1891817

14. Chen KJ, Lin SZ, Zhou L, Xie HY, Zhou WH, Taki-Eldin A, et al. Selective Recruitment of Regulatory T Cell Through CCR6-CCL20 in Hepatocellular Carcinoma Fosters Tumor Progression and Predicts Poor Prognosis. PloS One (2011) 6(9):e24671. doi: 10.1371/journal.pone.0024671

15. Arihara F, Mizukoshi E, Kitahara M, Takata Y, Arai K, Yamashita T, et al. Increase in CD14+HLA-DR -/Low Myeloid-Derived Suppressor Cells in Hepatocellular Carcinoma Patients and Its Impact on Prognosis. Cancer Immunol Immunother CII (2013) 62(8):1421-30. doi: 10.1007/s00262-013-1447-1

16. Han Y, Chen Z, Yang Y, Jiang Z, Gu Y, Liu Y, et al. Human CD14+ CTLA-4+ Regulatory Dendritic Cells Suppress T-Cell Response by Cytotoxic TLymphocyte Antigen-4-Dependent IL-10 and Indoleamine-2,3-Dioxygenase Production in Hepatocellular Carcinoma. Hepatol (Baltimore Md) (2014) 59 (2):567-79. doi: 10.1002/hep.26694

17. Sun JC, Lanier LL. NK Cell Development, Homeostasis and Function: Parallels With CD8+ T Cells. Nat Rev Immunol (2011) 11(10):645-57. doi: $10.1038 /$ nri3044

18. Llovet JM, Kelley RK, Villanueva A, Singal AG, Pikarsky E, Roayaie S, et al. Hepatocellular Carcinoma. Nat Rev Dis Primers (2021) 7(1):6. doi: 10.1038/ s41572-020-00240-3

19. Ishida Y, Agata Y, Shibahara K, Honjo T. Induced Expression of PD-1, a Novel Member of the Immunoglobulin Gene Superfamily, Upon Programmed Cell Death. EMBO J (1992) 11(11):3887-95. doi: 10.1002/ j.1460-2075.1992.tb05481.x

20. Krummel MF, Allison JP. CD28 and CTLA-4 Have Opposing Effects on the Response of T Cells to Stimulation. J Exp Med (1995) 182(2):459-65. doi: 10.1084/jem.182.2.459

21. Sharma P, Allison JP. The Future of Immune Checkpoint Therapy. Sci (New York NY) (2015) 348(6230):56-61. doi: 10.1126/science.aaa8172

22. Allison JP, Honjo T. The 2018 Nobel Prize in Physiology or Medicine. The Nobel Assembly at Karolinska Institutet Stockholm, Sweden (2018-10-01). Available at: https://www.nobelprize.org/prizes/medicine/2018/press-release/.

23. Jiang Y, Li Y, Zhu B. T-Cell Exhaustion in the Tumor Microenvironment. Cell Death Dis (2015) 6:e1792. doi: 10.1038/cddis.2015.162

24. Shi L, Chen S, Yang L, Li Y. The Role of PD-1 and PD-L1 in T-Cell Immune Suppression in Patients With Hematological Malignancies. J Hematol Oncol (2013) 6(1):74-. doi: 10.1186/1756-8722-6-74
25. Dai S, Jia R, Zhang X, Fang Q, Huang L. The PD-1/PD-Ls Pathway and Autoimmune Diseases. Cell Immunol (2014) 290(1):72-9. doi: 10.1016/ j.cellimm.2014.05.006

26. El-Khoueiry AB, Sangro B, Yau T, Crocenzi TS, Kudo M, Hsu C, et al. Nivolumab in Patients With Advanced Hepatocellular Carcinoma (CheckMate 040): An Open-Label, Non-Comparative, Phase 1/2 Dose Escalation and Expansion Trial. Lancet (London England) (2017) 389 (10088):2492-502. doi: 10.1016/s0140-6736(17)31046-2

27. Yau T, Park J, Finn R, Cheng A-L, Mathurin P, Edeline J, et al. CheckMate 459: A Randomized, Multi-Center Phase III Study of Nivolumab (NIVO) vs Sorafenib (SOR) as First-Line (1L) Treatment in Patients (Pts) With Advanced Hepatocellular Carcinoma (aHCC). Ann Oncol (2019) 30:v874v5. doi: 10.1093/annonc/mdz394.029

28. Zhu AX, Finn RS, Edeline J, Cattan S, Ogasawara S, Palmer D, et al. Pembrolizumab in Patients With Advanced Hepatocellular Carcinoma Previously Treated With Sorafenib (KEYNOTE-224): A Non-Randomised, Open-Label Phase 2 Trial. Lancet Oncol (2018) 19(7):940-52. doi: 10.1016/ S1470-2045(18)30351-6

29. Finn RS, Ryoo B-Y, Merle P, Bouattour M, Lim H, Breder V, et al. Pembrolizumab as Second-Line Therapy in Patients With Advanced Hepatocellular Carcinoma in KEYNOTE-240: A Randomized, Double-Blind, Phase III Trial. J Clin Oncol (2020) 3:193-202. doi: 10.1200/JCO.19.01307

30. Yang H, Shen K, Zhu C, Li Q, Zhao Y, Ma X. Safety and Efficacy of Durvalumab (MEDI4736) in Various Solid Tumors. Drug Design Dev Ther (2018) 12:2085-96. doi: 10.2147/DDDT.S162214

31. Lee D-W, Cho EJ, Lee J-H, Yu SJ, Kim YJ, Yoon J-H, et al. Phase II Study of Avelumab in Patients With Advanced Hepatocellular Carcinoma Previously Treated With Sorafenib. Clin Cancer Res (2021) 27(3):713-8. doi: 10.1158/ 1078-0432.CCR-20-3094

32. Vesely MD, Kershaw MH, Schreiber RD, Smyth MJ. Natural Innate and Adaptive Immunity to Cancer. Annu Rev Immunol (2011) 29:235-71. doi: 10.1146/annurev-immunol-031210-101324

33. Sangro B, Gomez-Martin C, de la Mata M, Inarrairaegui M, Garralda E, Barrera P, et al. A Clinical Trial of CTLA-4 Blockade With Tremelimumab in Patients With Hepatocellular Carcinoma and Chronic Hepatitis C. J Hepatol (2013) 59(1):81-8. doi: 10.1016/j.jhep.2013.02.022

34. Kandalaft LE, Motz GT, Busch J, Coukos G. Angiogenesis and the Tumor Vasculature as Antitumor Immune Modulators: The Role of Vascular Endothelial Growth Factor and Endothelin. Curr Top Microbiol Immunol (2011) 344:129-48. doi: 10.1007/82_2010_95

35. Hegde PS, Wallin JJ, Mancao C. Predictive Markers of Anti-VEGF and Emerging Role of Angiogenesis Inhibitors as Immunotherapeutics. Semin Cancer Biol (2018) 52(Pt 2):117-24. doi: 10.1016/j.semcancer.2017.12.002

36. Ohm JE, Gabrilovich DI, Sempowski GD, Kisseleva E, Parman KS, Nadaf S, et al. VEGF Inhibits T-Cell Development and may Contribute to TumorInduced Immune Suppression. Blood (2003) 101(12):4878-86. doi: 10.1182/ blood-2002-07-1956

37. Voron T, Colussi O, Marcheteau E, Pernot S, Nizard M, Pointet AL, et al. VEGF-A Modulates Expression of Inhibitory Checkpoints on CD8+ T Cells in Tumors. J Exp Med (2015) 212(2):139-48. doi: 10.1084/jem.20140559

38. Nuti M, Zizzari IG, Botticelli A, Rughetti A, Marchetti P. The Ambitious Role of Anti Angiogenesis Molecules: Turning a Cold Tumor Into a Hot One. Cancer Treat Rev (2018) 70:41-6. doi: 10.1016/j.ctrv.2018.07.016

39. Kwilas AR, Ardiani A, Donahue RN, Aftab DT, Hodge JW. Dual Effects of a Targeted Small-Molecule Inhibitor (Cabozantinib) on Immune-Mediated Killing of Tumor Cells and Immune Tumor Microenvironment Permissiveness When Combined With a Cancer Vaccine. J Transl Med (2014) 12:294. doi: 10.1186/s12967-014-0294-y

40. Kato Y, Tabata K, Kimura T, Yachie-Kinoshita A, Ozawa Y, Yamada K, et al. Lenvatinib Plus Anti-PD-1 Antibody Combination Treatment Activates CD8+ T Cells Through Reduction of Tumor-Associated Macrophage and Activation of the Interferon Pathway. PloS One (2019) 14(2):e0212513. doi: 10.1371/journal.pone. 0212513

41. Finn RS, Qin S, Ikeda M, Galle PR, Ducreux M, Kim TY, et al. Atezolizumab Plus Bevacizumab in Unresectable Hepatocellular Carcinoma. $N$ Engl J Med (2020) 382(20):1894-905. doi: 10.1056/NEJMoa1915745

42. Larkin J, Chiarion-Sileni V, Gonzalez R, Grob JJ, Cowey CL, Lao CD, et al. Combined Nivolumab and Ipilimumab or Monotherapy in Untreated 
Melanoma. New Engl J Med (2015) 373(1):23-34. doi: 10.1056/ NEJMoa1504030

43. Postow MA, Chesney J, Pavlick AC, Robert C, Grossmann K, McDermott D, et al. Nivolumab and Ipilimumab Versus Ipilimumab in Untreated Melanoma. New Engl J Med (2015) 372(21):2006-17. doi: 10.1056/NEJMoa1414428

44. Kudo M. Molecular Targeted Agents for Hepatocellular Carcinoma: Current Status and Future Perspectives. Liver Cancer (2017) 6(2):101-12. doi: 10.1159/ 000452138

45. He AR, Yau T, Hsu C, Kang Y-K, Kim T-Y, Santoro A, et al. Nivolumab (NIVO) + Ipilimumab (IPI) Combination Therapy in Patients (Pts) With Advanced Hepatocellular Carcinoma (aHCC): Subgroup Analyses From CheckMate 040. J Clin Oncol (2020) 38(4_suppl):512. doi: 10.1200/JCO.2020.38.4_suppl.512

46. Yau T, Kang Y-K, Kim T-Y, El-Khoueiry AB, Santoro A, Sangro B, et al. Efficacy and Safety of Nivolumab Plus Ipilimumab in Patients With Advanced Hepatocellular Carcinoma Previously Treated With Sorafenib: The CheckMate 040 Randomized Clinical Trial. JAMA Oncol (2020) 6(11): e204564-e. doi: 10.1001/jamaoncol.2020.4564

47. Kelley RK, Abou-Alfa GK, Bendell JC, Kim T-Y, Borad MJ, Yong W-P, et al. Phase I/II Study of Durvalumab and Tremelimumab in Patients With Unresectable Hepatocellular Carcinoma (HCC): Phase I Safety and Efficacy Analyses. J Clin Oncol (2017) 35(15_suppl):4073. doi: 10.1200/ JCO.2017.35.15_suppl.4073

48. Kudo M, Motomura K, Wada Y, Inaba Y, Sakamoto Y, Kurosaki M, et al. Avelumab in Combination With Axitinib as First-Line Treatment in Patients With Advanced Hepatocellular Carcinoma: Results From the Phase 1b VEGF Liver 100 Trial. Liver Cancer (2021) 10(3):249-59. doi: 10.1159/000514420

49. Kato Y, Bao X, Macgrath S, Tabata K, Hori Y, Tachino S, et al. Lenvatinib Mesilate (LEN) Enhanced Antitumor Activity of a PD-1 Blockade Agent by Potentiating Th1 Immune Response. Ann Oncol (2016) 27(suppl_6):1-14. doi: $10.1093 / \mathrm{annonc} / \mathrm{mdw} 362.02$

50. Kudo M. Immune Checkpoint Inhibition in Hepatocellular Carcinoma: Basics and Ongoing Clinical Trials. Oncology (2017) 92 Suppl 1:50-62. doi: 10.1159/ 000451016

51. Brahmer JR, Lacchetti C, Schneider BJ, Atkins MB, Brassil KJ, Caterino JM, et al. Management of Immune-Related Adverse Events in Patients Treated With Immune Checkpoint Inhibitor Therapy: American Society of Clinical Oncology Clinical Practice Guideline. J Clin Oncol (2018) 36(17):1714-68. doi: 10.1200/JCO.2017.77.6385

52. Ramnaraign BH, Chatzkel JA, Al-Mansour ZA, Rogers S, Jones D, DeRemer D, et al. Immunotherapy Management in Special Cancer Patient Populations. JCO Oncol Pract (2021) 17(5):240-5. doi: 10.1200/OP.20.00996

53. Elsegood CL, Tirnitz-Parker JE, Olynyk JK, Yeoh GC. Immune Checkpoint Inhibition: Prospects for Prevention and Therapy of Hepatocellular Carcinoma. Clin Trans Immunol (2017) 6(11):e161. doi: 10.1038/cti.2017.47

54. Wolchok JD, Chiarion-Sileni V, Gonzalez R, Rutkowski P, Grob JJ, Cowey CL, et al. Overall Survival With Combined Nivolumab and Ipilimumab in
Advanced Melanoma. N Engl J Med (2017) 377(14):1345-56. doi: 10.1056/ NEJMoa1709684

55. Hodi FS, Chesney J, Pavlick AC, Robert C, Grossmann KF, McDermott DF, et al. Combined Nivolumab and Ipilimumab Versus Ipilimumab Alone in Patients With Advanced Melanoma: 2-Year Overall Survival Outcomes in a Multicentre, Randomised, Controlled, Phase 2 Trial. Lancet Oncol (2016) 17 (11):1558-68. doi: 10.1016/S1470-2045(16)30366-7

56. Hellmann MD, Paz-Ares L, Bernabe Caro R, Zurawski B, Kim SW, Carcereny Costa E, et al. Nivolumab Plus Ipilimumab in Advanced Non-Small-Cell Lung Cancer. N Engl J Med (2019) 381(21):2020-31. doi: 10.1056/NEJMoa1910231

57. Yau T, Kang YK, Kim TY, El-Khoueiry AB, Santoro A, Sangro B, et al. Efficacy and Safety of Nivolumab Plus Ipilimumab in Patients With Advanced Hepatocellular Carcinoma Previously Treated With Sorafenib: The CheckMate 040 Randomized Clinical Trial. JAMA Oncol (2020) 6(11): e204564. doi: 10.1001/jamaoncol.2020.4564

58. Kelley RK, Sangro B, Harris W, Ikeda M, Okusaka T, Kang YK, et al. Safety, Efficacy, and Pharmacodynamics of Tremelimumab Plus Durvalumab for Patients With Unresectable Hepatocellular Carcinoma: Randomized Expansion of a Phase I/II Study. J Clin Oncol (2021) 39(27):2991-3001. doi: $10.1200 /$ JCO.20.03555

59. Garralda E, Sukari A, Lakhani NJ, Patnaik A, Lou Y, Im S, et al. A Phase 1 First-in-Human Study of the Anti-LAG-3 Antibody MK4280 (Favezelimab) Plus Pembrolizumab in Previously Treated, Advanced Microsatellite Stable Colorectal Cancer. J Clin Oncol (2021) 39:3584-4. doi: 10.1200/JCO.2021. 39.15_suppl.3584

60. Thompson JA, Schneider BJ, Brahmer J, Andrews S, Armand P, Bhatia S, et al. NCCN Guidelines Insights: Management of Immunotherapy-Related Toxicities, Version 1.2020. J Natl Compr Canc Netw (2020) 18(3):230-41. doi: $10.6004 /$ incen.2020.0012

Conflict of Interest: The authors declare that the research was conducted in the absence of any commercial or financial relationships that could be construed as a potential conflict of interest.

Publisher's Note: All claims expressed in this article are solely those of the authors and do not necessarily represent those of their affiliated organizations, or those of the publisher, the editors and the reviewers. Any product that may be evaluated in this article, or claim that may be made by its manufacturer, is not guaranteed or endorsed by the publisher.

Copyright (c) 2021 Ozer, George, Goksu, George and Sahin. This is an open-access article distributed under the terms of the Creative Commons Attribution License (CC BY). The use, distribution or reproduction in other forums is permitted, provided the original author(s) and the copyright owner(s) are credited and that the original publication in this journal is cited, in accordance with accepted academic practice. No use, distribution or reproduction is permitted which does not comply with these terms. 